\title{
2015 Laboratory Directed Research Fermilab \& Development Program Plan
}
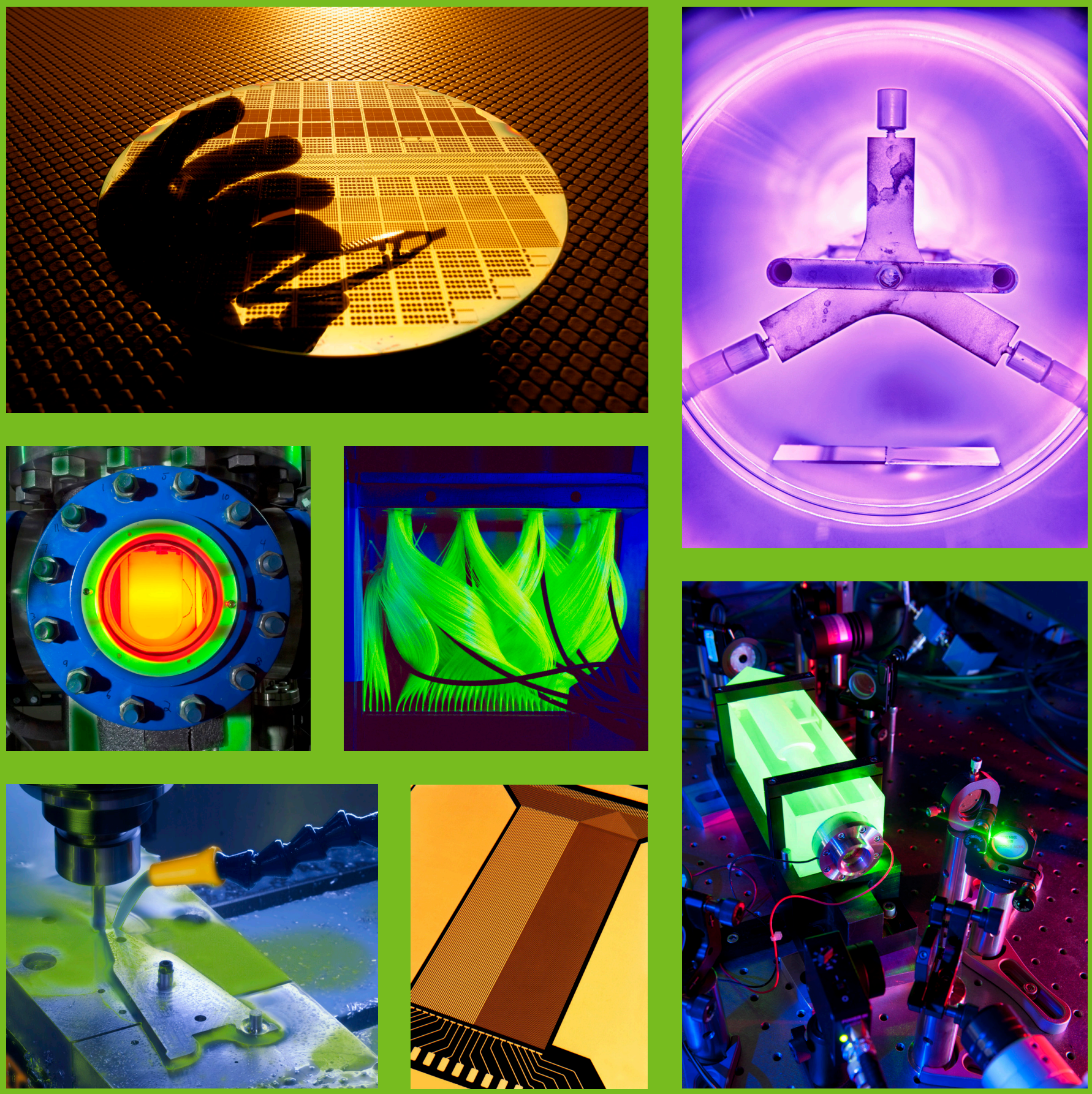

* Fermilab

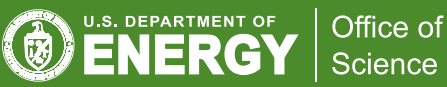


Table of Contents

LIST OF ACRONYMS USED................................................................................ iii

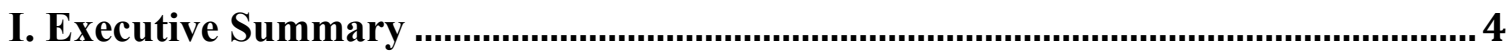

II. Changes from previous year Fermilab LDRD Annual Program Plan.................... 5

III. LDRD and Laboratory and Agency Mission ............................................................. 6

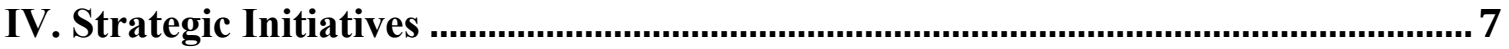

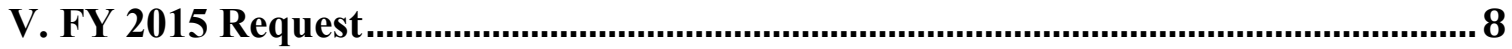

VI. LDRD General Description ................................................................................ 8

VII. LDRD Management and Administration ........................................................ 10

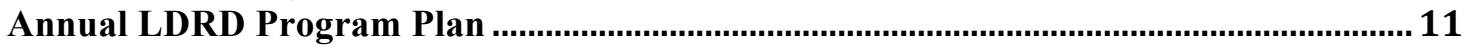

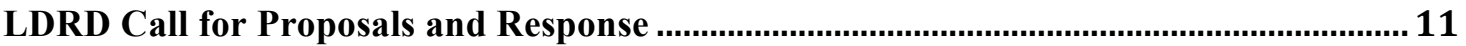

Ongoing LDRD Projects Seeking $2^{\text {nd }}$ or $3^{\text {rd }}$ Year Funding ............................................... 12

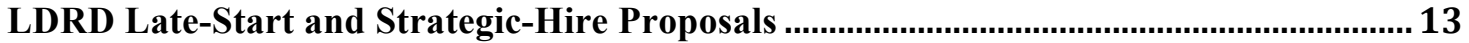

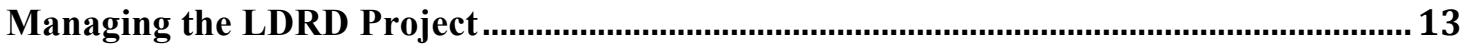

LDRD Reporting 


\section{LIST OF ACRONYMS USED}

$\begin{array}{ll}\text { CAO } & \text { Chief Accelerator Officer } \\ \text { CAS } & \text { Contractor Assurance System } \\ \text { CFO } & \text { Chief Financial Officer } \\ \text { CIO } & \text { Chief Information Officer } \\ \text { COO } & \text { Chief Operating Officer } \\ \text { CPO } & \text { Chief Program Officer } \\ \text { CRO } & \text { Chief Research Officer } \\ \text { CSO } & \text { Cognizant Secretarial Officer } \\ \text { CTO } & \text { Chief Technology Officer } \\ \text { DOE } & \text { Department of Energy } \\ \text { D/S/C } & \text { Division / Section / Center } \\ \text { EO } & \text { Environmental Officer } \\ \text { FFM } & \text { Field Financial Manager } \\ \text { FNAL } & \text { Fermi National Accelerator Laboratory } \\ \text { FSO } & \text { Fermi Site Office } \\ \text { FY } & \text { Fiscal Year } \\ \text { LDRD } & \text { Laboratory Directed Research and Development } \\ \text { NEPA } & \text { National Environmental Protection Act } \\ \text { NNSA } & \text { National Nuclear Security Administration } \\ \text { OHEP } & \text { Office of High Energy Physics } \\ \text { OSTI } & \text { Office of Science and Technical Information } \\ \text { PI } & \text { Principal Investigator } \\ \text { SO } & \text { Safety Officier } \\ & \end{array}$




\section{Executive Summary}

Fermilab is executing Laboratory Directed Research and Development (LDRD) as outlined by order DOE O 413.2B in order to enhance and realize the mission of the laboratory in a manner that also supports the laboratory's strategic objectives and the mission of the Department of Energy. LDRD funds enable scientific creativity, allow for exploration of "high risk, high payoff" research, and allow for the demonstration of new ideas, technical concepts, and devices. LDRD also has an objective of maintaining and enhancing the scientific and technical vitality of Fermilab.

LDRD is able to fund employee-initiated proposals that address the current strategic objectives and better position Fermilab for future mission needs. The request for such funds is made in consideration of the investment needs, affordability, and directives from DOE and Congress. Review procedures of the proposals will ensure that those proposals which most address the strategic goals of the DOE and the Laboratory or which best position Fermilab for the future will be recommended to the Laboratory Director who has responsibility for approval. The execution of each approved project will be the responsibility of the Principal Investigator, PI, who will follow existing Laboratory guidelines to ensure compliance with safety, environmental, and quality assurance practices. A Laboratory Director-appointed LDRD Coordinator will work with Committees, Laboratory Management, other Fermilab Staff, and the PI's to oversee the implementation of policies and procedures of LDRD and provide the management and execution of this Annual Program Plan.

FY14 represented the first year for LDRD at Fermilab. In FY14, Fifty (50) preliminary proposals and 29 full proposals were submitted indicating very strong interest of the program within the Fermilab community. Seven LDRD projects have been selected and are now being executed. The implementation of the program compared with FY14 is mostly unchanged except that the roles and responsibilities of senior laboratory management has been updated to reflect reorganization within the senior management (Director, Deputy Director, CRO, Deputy CRO, COO, CFO, CAO, CIO, CPO, CTO, and $\mathrm{D} / \mathrm{S} / \mathrm{C}$ heads.

In FY 2015, the Fermi National Accelerator Laboratory requests that approval be granted for an LDRD expenditure comprising up to 1.0\% (\$3.5M) of the laboratory's total operating / capital equipment budget. 


\section{Changes from previous year Fermilab LDRD Annual Program Plan}

References to the "three frontiers" of particles physics: Intensity, Energy, and Cosmic have been modified to make reference to the "five science drivers" as outlined by the P5 report approved by the High Energy Physics Advisory Committee.

References throughout this document to the "initial year of LDRD at Fermilab" have been modified to reflect the current fiscal year and the experience gained in FY14.

References to roles and responsibilities of the Associate Laboratory Directors have been changed to reflect the reorganization within the senior Laboratory Management.

The request for FY15 of \$3.5M is an increase over FY14 to continue funding LDRD projects approved in FY14 and to allow for approximately $\$ 1 \mathrm{M}$ of funding for new projects starting in FY15.

Approximate dates are established for the FY15 implementation.

Sep 2014: On-going projects provide a status report and suggest modifications, if any, to the Project Data Sheet

Oct 2014: Call for Proposals

Oct 2014: Preliminary Proposals due

Nov 2014: Full Proposals due

Dec 2014: Mid-year Progress Reviews of FY14 Projects

Dec 2014: Selection Committee Review of FY15 Proposals

Jan 2015: Start of FY15 LDRD Projects

Apr 2015: estimated date for Call for FY16 Proposals with start in Oct 2015 


\section{LDRD and Laboratory and Agency Mission}

\section{Department of Energy Mission}

The mission of the Energy Department is to ensure America's security and prosperity by addressing its energy, environmental and nuclear challenges through transformative science and technology solutions.

$\underline{\text { Fermilab Mission }}$

Our mission is to drive discovery in particle physics by

- building and operating world-leading accelerator and detector facilities

- performing pioneering research with global partners

- transforming technologies for science and industry

The Mission statements of Fermilab reflect the pursuit of excellence in scientific research in the area of particle physics. Particle physics addresses scientific mysteries in matter, energy, space and time through five science drivers as enabled by theoretical, computational, accelerator, instrumentation and other technical capabilities. The five science drivers are to 1) use the Higgs boson as a new tool for discovery, 2) pursue the physics associated with the neutrino mass, 3) identify the new physics of dark matter, 4) understand cosmic acceleration: dark energy and inflation, and 5) explore the unknown: new particles, interactions, and physical principles. Fermilab's Mission statement reflects Fermilab's role in support of the overall mission of the Department of Energy. In particular, "transformative science and technology solutions" in the area of particle physics will be furthered through the use of LDRD where flexibility and efficiency will enable investigators to carry out creative new projects in forefront areas that enrich the current Fermilab program and strategically put Fermilab in a better position to deliver the mission objectives of DOE and Fermilab for the future. 


\section{Strategic Initiatives}

The strategic objectives of Fermilab are to establish excellence in Particle Physics, Accelerator Science and Technology, and Large-Scale User Facilities. As a facility for worldwide Users, Fermilab provides a complex that safely and efficiently delivers the highest levels of performance, provides theoretical insights, and the development of new technologies. World-class scientific, engineering, computing, and support staff use wellintegrated, efficient, business and management systems to operate Fermilab and its associated infrastructure. Providing Fermilab employees the opportunity to think creatively in terms of proposing LDRD projects and then executing those projects will likely lead to many cases where the new ideas become a seed for further investigation in the broader scope of what Fermilab can offer to DOE and its users. Beyond the immediate impact upon Fermilab and the mission of DOE, certain projects may have secondary impact on national priorities relevant to other federal agencies.

As LDRD allows for support of higher risk proposals that have the potential for higher payoff, it is not known which projects will emerge as having the greatest lasting impact. It is understood that exploratory research may result in a range of possible outcomes. Even in those cases where the result is less than desired, there may be seeds for new ideas that later become fruitful. The very process of carrying out an LDRD project will benefit the development of the Principal Investigator (PI) professionally and have a lasting impact on their ability to carry out future laboratory work. LDRD provides a flexibility that will allow for the most promising proposals to get funded and allow the investigators to see their new ideas to completion.

For this second year of LDRD, the strategic focus again will be to solicit a number of small and modest scale proposals from a broad cross section of the laboratory's staff. The FY15 Call for Proposals will emphasize and encourage a broad scope of proposals. The proposals are expected to be of a type that will extend the current programmatic areas with the possibility of advancing into new emerging areas of laboratory focus. The Laboratory Director sets this strategic focus after receiving input from senior laboratory management and the LDRD Advisory Committee, which is comprised of individuals representing many sectors of the Laboratory's scientific and technical areas. 


\section{FY 2015 Request}

In FY 2015, the Fermi National Accelerator Laboratory requests that approval be granted for an LDRD expenditure comprising up to $1.0 \%(\$ 3.5 \mathrm{M})$ of the laboratory's total operating / capital equipment budget.

The annual LDRD budget is developed after careful consideration of investment needs, affordability in our indirect budgets, directives from DOE and Congress, and compliance with order DOE 413.2B governing the LDRD program. This total request reflects that FY 2015 represents a second year of the LDRD program at Fermilab and that the FY 2015 program will be conducted during the second half of FY 2015. The amount requested represents the best judgment to balance the LDRD program with the funding required to meet the current programmatic deliverables and to meet the varied demands on the laboratory overhead. The request will enable the FY 2015 LDRD program to continue funding for existing LDRD projects and to allow for the selection of another set of proposals that should realize both the specific and broader objectives of the LDRD program.

\section{LDRD General Description}

Fermilab's Laboratory Directed Research and Development seeks to fund employee initiated proposals in research and development in science and technology areas to maintain the scientific and technical vitality of the laboratory, enhance Fermilab's ability to realize its own and DOE's mission, foster creativity and exploration in forefront areas, serve as a proving ground for new concepts, and provide a means for which high-risk and potentially high-value research and development can be carried out. The program will encourage employees to think creatively and to pursue ideas in areas that support and potentially enhance the scientific and technical capabilities of the laboratory. LDRD will highlight the overall missions of the laboratory and DOE with a focus that encourages new innovative approaches to technical and scientific problems. The program will encourage exploration in new areas that may impact areas of future focus. LDRD will provide a way to initiate new ideas and perform preliminary technical assessments to determine "proof of principle" or early determination of the utility of the new idea, concept, or device. The program will facilitate the possibility of breakthrough developments in science in technology when a high-risk idea is successful. Even when the research and development reaches less desired outcomes, LDRD will provide benefits to the investigator's professional development and the laboratory's scientific and technical vitality.

The Fermilab LDRD program solicits employee proposals for research and development that are of relatively small scale both in cost and duration and yet score high on measures of excellence in terms of innovation in forefront areas of science and technology. The proposals can include experiments, theoretical studies, and simulations. Proposals can include technological development directed towards an early determination of the utility 
of the developed idea, technology, devices, or research tools. Proposals can be focused on new concepts and preliminary technical analysis of the advanced, novel experimental facility or device.

Proposals that are submitted will be evaluated on criteria that emphasize excellence in both scientific and technical merit and strategic merit. The scientific and technical significance will be judged on the importance of the proposed work to advance knowledge and understanding both within high energy physics and potentially across different fields. The proposals will also be evaluated on the innovativeness and whether the novel concepts are to be explored. In addition, the PI and others involved in the project will be evaluated on the basis of the PI's qualifications and whether the required expertise is sufficient to address the technical requirements of the proposed research plan. The proposal is expected to be of sufficient quality in clearly defining the proposed activity and making credible estimates of time and effort so that the proposed project is likely to be successfully completed within the scope of funding levels and duration. Proposals will also be evaluated in terms of the overall relevance to the missions both of the DOE and of Fermilab. The proposal will be evaluated as to its relevance of the objectives of LDRD and meeting the Laboratory's strategic goals. The evaluation process will also determine whether the proposed research and development will be likely to have enduring benefits and whether the project enhances the Laboratory's reputation in the scientific and technical community.

Under DOE O 413.2B, proposals that are funded under LDRD will also be selected only if the proposal is not already part of the programmatic portfolio of the Laboratory. In addition, each proposal is required to be completed only with LDRD funds and not supplemented with non-LDRD funds. Proposals can be for preliminary studies but cannot be used to fund construction projects or design beyond the preliminary phase. Capital expenditures are allowed for general purpose equipment that is not otherwise readily available from the Laboratory inventory.

In short, Fermilab's implementation of LDRD will address the overall objectives of the program toward providing support of research and development in novel and innovative areas where new concepts can be explored. At the same time, the presence of the program will maintain the scientific and technical vitality of the Laboratory. The proposals that will be funded will be those that demonstrate both scientific and technical merit as well as strategic merit. Successful proposals can result in new areas of exploration within Fermilab and enhance the Laboratory's and DOE's missions of pursuing scientific excellence. 


\section{LDRD Management and Administration}

With the guidance from DOE Order 413.2B and a DOE "Roles, Responsibilities, and Guidelines for LDRD at the DOE/NNSA Laboratories" guidance document dated 6/13/06, a committee of scientists, engineers, and managers met as the LDRD Advisory Committee to provide specific implementation guidance for LDRD at Fermilab. Input from this Advisory Committee and FNAL senior laboratory management along with experience obtained during the initial FY14 implementation of the program has been incorporated into this Annual Plan. Additionally, insights obtained from attending the LDRD Review at SLAC and LBNL have also been incorporated in the Annual LDRD Program Plan. In addition to this Annual LDRD Program Plan, a set of controlled documents describing the implementation of the Fermilab LDRD program is maintained. The LDRD Coordinator maintains these documents under the Science Management (M4) framework of the Contractor Assurance System (CAS). The LDRD Coordinator also chairs and acts as the Recording Secretary of the LDRD Advisory Committee and is an ex officio member of the LDRD Selection Committee.

The Laboratory Director is responsible for establishing the annual budget for LDRD and for setting priorities for strategic areas that will be considered by the LDRD Selection Committee. The Director appoints members of both the LDRD Advisory and Selection Committees. The Laboratory Director approves this Annual LDRD Program Plan and secures approval from DOE. The Laboratory Director is responsible for approving the final list of projects recommended to be funded and securing Fermi Site Office (FSO) concurrence of those projects.

The Chief Operating Officer (COO) with the Chief Financial Officer (CFO) have oversight responsibility for the Fermilab overhead budget from which LDRD is funded. The senior Laboratory management including the Chief Research Officer and Deputy, Chief Technology Officer, Chief Accelerator Officer, Chief Information Officer, and Chief Project Officer recommend topics for the annual LDRD Call for Proposals and nominate members for the LDRD Advisory and Selection Committees.

The Division, Sector, Center $(\mathrm{D} / \mathrm{S} / \mathrm{C})$ Heads provide oversight prior to the submission of both the preliminary and full proposals. This oversight should be done in consultation with other managers in the $\mathrm{D} / \mathrm{S} / \mathrm{C}$ organization along with the Supervisor of the Principal Investigator to make sure the PI is able to conduct the proposed work within the context of the PI's programmatic responsibilities and to ensure that requested resources are likely to be available for the work under the proposal. D/S/C Heads also nominate employees to serve as members of the LDRD Selection Committee.

The LDRD Selection Committee reviews and recommends both preliminary and full proposals. The LDRD Selection Committee recommends projects to be funded to the Laboratory Director. The Selection Committee also reviews progress reports and makes recommendations regarding $2^{\text {nd }}$ and $3^{\text {rd }}$ year funding for multi-year LDRD projects. 
The Principal Investigator prepares the preliminary and full proposals and contributes to a Project Data Sheet. The PI is responsible for managing the LDRD funded project within budget and schedule constraints and for managing the project following established Laboratory guidelines including adhering to environmental, safety, and quality assurance policies and working with D/S/C Environmental (EO) and Safety (SO) Officers. The PI will work with D/S/C Field Financial Managers (FFMs) to develop the budget estimates and to obtain project and task codes once approved. The PI is responsible for reporting the results of the investigation and preparing a required year-end Project Summary report.

\section{Annual LDRD Program Plan}

The Annual LDRD Program Plan is a required document, which is updated each fiscal year and which serves as the approval document by DOE and the Laboratory for carrying out the LDRD program annually. The LDRD Coordinator has responsibility for producing this document after taking input from the Laboratory Director, Senior Laboratory Management, D/S/C Heads, and the LDRD Advisory Committee. The CFO also plays a role in reviewing the draft Annual LDRD Program Plan in order to identify any possible budget issues and to provide a recommended budget level. The Laboratory Director approves a final version of the document and ensures that it is forwarded to the DOE for approval.

The Laboratory Director, in particular, provides high-level guidance on the strategic priorities to be emphasized in the Call for Proposals including whether an amount should be set aside for Late-Start or Strategic-Hire proposals.

This Annual LDRD Program Plan incorporates a number of elements including a one page Executive Summary that describes the objectives of the LDRD program, the level of funding being requested, and a statement of justification for that funding level in terms of the benefit to DOE and Fermilab in terms of meeting the broad mission statements of the DOE and Fermilab. The Annual LDRD Program Plan highlights the strategic priorities that the Laboratory Director wishes to emphasize.

The Plan also provides additional detail of the general description and justification of the LDRD program including the objectives, characteristics and selection criteria for LDRD Projects. The document also outlines the plan for LDRD Management and Administration. In short, the Annual LDRD Program Plan is a statement of the Laboratory's planned implementation of the LDRD program for the fiscal year.

\section{LDRD Call for Proposals and Response}

The Call for Proposals in FY2015 will be made during October 2014. The LDRD Call for Proposals will require potential PI's to submit a Preliminary Proposal along with a Full Proposal. The Call for Proposals will be advertised throughout the Laboratory in order to engage a broad spectrum of employees. 
The purpose of the Preliminary Proposal is for the PI to have a discussion with the PI's line management Supervisor and the appropriate Division/Sector/Center Head to determine whether there would be any issues with the PI being able to execute the project work plan. The Preliminary Proposal is meant to be a very short document to facilitate this discussion. The Preliminary Proposal consists of a brief Project Summary and Proposed Project Work Plan. By involving the line management Supervisor and the $\mathrm{D} / \mathrm{S} / \mathrm{C}$ Head early, it is hoped that potential issues dealing with the proposed amount of time and other resources that the PI is expecting to spend on the LDRD project can be resolved within the organization. The LDRD Selection Committee also reviews the Preliminary Proposal and feedback and guidance is provided to the PI. Based upon this feedback, the PI may or may not be encouraged to continue and submit a Full Proposal.

The Full Proposal will be a document submitted by the PI based upon a template example, made available by the LDRD coordinator. The Full Proposal will provide a Project Title and identify the Principal Investigator. The Full Proposal will list any coinvestigators including additional Fermilab staff members who will charge time towards the LDRD Project along with University researchers who will collaborate on the project. The Full Proposal will have a description the proposed fiscal-year and total budgets that include fully burdened personnel costs along with required and allowable materials and service costs. The budget information will be prepared with cooperation of $\mathrm{D} / \mathrm{S} / \mathrm{C}$ Field Financial Managers. The Proposal will consist of a summary Project Description and description of the significance of the proposed project. The core component of the Full Proposal will be a description of the proposed Research Plan with a clearly stated objective of the proposed research, a description of the scientific hypothesis or technical concept to be demonstrated, the methods, materials, facilities, and techniques that will be required to carry out the research. The expected results and impact of the research and development will also be described. An emphasis will be for Full Proposals to provide clear deliverables for each fiscal year. Preliminary and Full Proposals will be due in Oct/Nov 2014 respectively.

\section{Ongoing LDRD Projects Seeking $2^{\text {nd }}$ or $3^{\text {rd }}$ Year Funding}

For LDRD Projects that seek $2^{\text {nd }}$ or $3^{\text {rd }}$ Year Funding, it is understood that LDRD is funded out of Laboratory overhead and, as such, the availability of funds in $2^{\text {nd }}$ or $3^{\text {rd }}$ Year is contingent upon new fiscal year funding for LDRD. By the beginning of the fiscal year, each project seeking continued funding will be required to provide a written brief status report and to advise of any modifications to the Project Data Sheet. These reports and updated Project Data Sheets will be made available to the LDRD Selection Committee, the Director, and DOE to identify any issues prior to a mid-year Progress Review. Those LDRD Projects seeking $2^{\text {nd }}$ or $3^{\text {rd }}$ year funding will be required to make a presentation to the LDRD Selection Committee as a mid-year Progress Review. This review will allow for the Review Committee to hear a status report, ask questions, and deliberate. The LDRD Review Committee will recommend whether funding should be stopped or continued. If the project is recommended to be continued, the LDRD Review Committee also includes a recommendation as to whether the project should proceed in accordance with the original research plan or contingent upon specific changes. The LDRD Coordinator transmits the recommendation to the Laboratory Director for final 
approval. For projects funded mid-year in FY14, the mid-year Progress Review will occur in concert with the Dec 2014 review of new proposals submitted.

\section{LDRD Late-Start and Strategic-Hire Proposals}

In addition to the LDRD proposals received during the Call for Proposals, LDRD has a benefit to the Laboratory to allow for responding to exceptional opportunities that might arise during the fiscal year. For example, these opportunities may target a particular new strategic area. In the case of a Late-Start proposal received after the Call for Proposals, the LDRD Coordinator will obtain concurrence of the strategic importance of the proposal with the Laboratory Director and will obtain budgetary guidance with the Chief Financial Officer to determine whether funding will fit within the approved level of LDRD funding. The LDRD Coordinator will then convene the LDRD Selection Committee to evaluate the proposal as any other LDRD proposal. LDRD Proposals may also be used to initiate an opportunity for a Strategic-Hire to bring to the Laboratory new capabilities.

\section{Managing the LDRD Project}

The responsibility for managing the LDRD Project lies with the PI who is required to follow Laboratory policy and procedures to be in compliance with environmental, safety, and quality assurance practices. The PI will develop the detailed work plan for the project and secure necessary approvals from the environmental and safety officers as required. The PI will work with $\mathrm{D} / \mathrm{S} / \mathrm{C}$ Heads to ensure necessary resources are available to carry out the work plan. The PI will be able to begin work on an approved LDRD project after the project and task codes are in place. The PI is responsible for expenditures under those budgetary codes. The PI will be responsible for managing all expenditures associated with the approved project and following cost-effective and efficient use of approved funds. The PI will manage the technical scope and schedule in accordance with the detailed work plan. In executing the project, the PI will be able to secure materials, services, supplies, sub-contracts according to the work plan and in compliance with allowable LDRD expenditures. The PI is responsible for tracking and controlling costs.

The LDRD Coordinator will review LDRD expenditures on a monthly basis and be available to work with the PI and Field Financial Manager to resolve any issues in the event that the Project Plan requires any modification in scope or schedule. The proposal will be written in a manner that contains a detailed budget along with an expected level of contingency within which the project will be executed. If changes to the scope or schedule are small, it is expected that they can be accommodated within the level of contingency funding. If changes to the scope or schedule are sufficiently large, the LDRD Selection Committee will review the proposed changes and make a recommendation that requires approval by the Laboratory Director. In all cases, any modifications to the scope or schedule are required to fit within the allowable cost range as spelled out in the approved Annual LDRD Program Plan. 


\section{LDRD Reporting}

The DOE Order 413.2B requires a number of documents to be produced in association with the LDRD Program. The Annual LDRD Program Plan is one required document that requires approval each fiscal year as it spells out the requested funding level, provides a general description of the LDRD program and an explanation of how the LDRD program will meet the Laboratory needs and support the DOE and Laboratory mission.

In addition to the Annual LDRD Program Plan, there will be an LDRD Annual Report that provides a brief program overview and individual technical reports for each active and completed LDRD project. The Project Summaries of each LDRD project will include a general description of the objectives and purpose of the project along with a summary of scientific or technical progress achieve. The Project Summaries will also contain a brief statement as to how the project benefited the DOE and Laboratory missions. The PI has responsibility for writing each Project Summary. The LDRD Coordinator has the responsibility of compiling those summaries along with the other material into the LDRD Annual Report. This report will be provided to the Office of Scientific and Technical Information as required by DOE Order O 413.2B.

In addition to the required annual reporting, each approved project will have a Project Data Sheet prepared with the assistance of the PI to provide the FSO with sufficient background and technical information on which to base concurrence. These Project Data Sheets will provide the Laboratory name and fiscal year, a Project identifier, title, and the name of the PI. A short Project Description will explain the forefront scientific and technical aspects of the Project and a description of how the Project is tied to the mission. The Project Data Sheet will describe, if applicable, the previous year's accomplishments and the work proposed for the fiscal year.

The LDRD Coordinator will have responsibility for coordinating an annual LDRD Program Review with DOE to receive an evaluation on the compliance with DOE Order 413.2B, the LDRD program results, and the operation of the LDRD management system including the project selection process. In addition, the LDRD Coordinator will compile and make available annual performance indicators such as the numbers of graduate student and postdoctoral researchers supported under LDRD, the numbers of LDRDderived publications, invention disclosures, patents, and copyrights, and information on national awards and recognition received attributable in whole or in part to LDRD projects funded that fiscal year. The PI will provide these performance metrics to the LDRD Coordinator in an Annual LDRD Project Survey. 